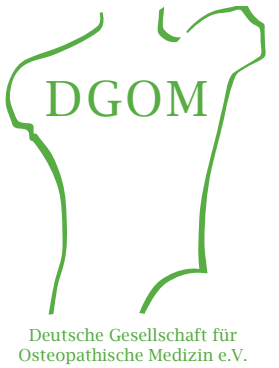

\title{
Deutsche Gesellschaft für Osteopathische Medizin (DGOM) e.V.
}

\author{
Information und Anmeldung: \\ Diakonie-Krankenhaus, Belchenstr. 1-5, 68163 Mannheim \\ Tel.: 0621/43626692, Fax: 0621/43626691 \\ Internet: kontakt@dgom.info,www.dgom.info
}

Leitung der DGOM-Akademie:

Dr. med. Volker Rings, Facharzt für Allgemeinmedizin, Manuelle Medizin, Osteopathische Medizin D.O.M. ${ }^{\mathrm{TM}}$

Teilnehmer: Ärzte und Physiotherapeuten mit Nachweis einer begonnenen manualmedizinischen Weiterbildung im Rahmen des Integrierten Curriculums oder einer abgeschlossenen Weiterbildung in Manueller Medizin bei einem der DGMM-Seminare bzw. einem anderen anerkannten Weiterbildungsträger.

Bitte beachten Sie auch das Curriculum der SAGOM.

$\begin{array}{ll}\begin{array}{l}\text { Grundcurriculum } \\ \text { GKOMT } \\ \text { 03.-05.07.2015 }\end{array} & \text { Freiburg } \\ \text { 10.-12.09.2015 } & \text { Mainz } \\ \text { 20.-22.11.2015 } & \text { Boppard } \\ \text { MET 1 } & \\ \text { 18.-20.09.2015 } & \text { Freiburg } \\ \text { MET 2 } & \\ \text { 13.-15.11.2015 } & \text { Freiburg } \\ \text { MET 3 } & \\ \text { 04.-06.12.2015 } & \text { Freiburg } \\ \text { MFR 1 } & \end{array}$

30.10.-01.11.2015 Hamburg

\section{MFR 2}

10.-12.07.2015 Berlin

25.-27.09.2015 Boppard (OV)*

Counterstrain

03.-05.07.2015 Boppard

Parietale Integration

11.-13.12.2015 Boppard (OV)*

Func 1

08.-10.05.2015 Lübeck

04.-06.12.2015 Boppard

Func 2

12.-14.06.2015 Mannheim

30.10.-01.11.2015 Boppard (OV)

Anatomie

18.-20.09.2015 Erlangen

06.-08.12.2015 Erlangen

Cranio 1

16.-18.10.2015 Mannheim

Cranio 2

17.-19.07.2015 Mannheim

11.-13.12.2015 Mainz

Cranio 3

03.-05.07.2015 Lübeck

27.-29.11.2015 Mannheim

Vis 1

23.-25.10.2015 Boppard
Vis 2

18.-20.09.2015 Hamburg

27.-29.11.2015 Mainz

Vis 3

10.-12.07.2015 Lübeck

04.-06.12.2015 Mannheim

Cranio-Refresher

09.-11.10.2015 Boppard

Masterkurse

Masterkurse allgemein

16.-18.10.2015 Boppard

Maurice César - Fascial Approach of the Patient 2

08.-11.10.2015 Freiburg

Marco Forte - Funktionelle Medizin nach Forte 2

27.-29.11.2015 Boppard

Rolf Hansen/Dietmar Hellmich - Das Kiefergelenk (TMG) 2

11.-13.09.2015 Mannheim

Boyd Buser - HVLA-Techniken

03.-05.07.2015 Hamburg

Thomas Seebeck, Theo Rudolf - Osteopathische Eigenübungen 25.-27.09.2015 Mainz

Ruppert Wellstein - Manuelle Thermodiagnostik und -therapie

02.-04.10.2015 Freiburg

Claudia Hafen - Mechanical Link 6 (nach P. Chauffour)

27.-29.11.2015 Freiburg

Paul Chauffour - Mechanical Link 7

11.-13.12.2015 Freiburg

Markus Erhard - Anatomy Trains - Myofasciales Taping 2

01.-04.11.2015 Münster

Ken Lossing - Viscerale Lymphdrainage

Masterkursreihe Osteopathische Pädiatrie

13.-15.11.2015 Mannheim

Jane Carreiro - Päd-Basiskurs 2 (BLT - Sutherland Approach)

Masterkursreihe Osteopathische Geriatrie

09.-11.10.2015 Mannheim

Johannes Mayer - Einführungskurs Osteopathische Geriatrie mit indirekten Techniken

23.-25.10.2015 Berlin

Johannes Mayer - Becker-Techniken u. Viscerale Osteopathie im Alter 3

Weitere Kurstermine für das Jahr 2015 befinden sich auf unserer

Homepage: www.dgom.info.

*OV: Osteopathische Verfahren

ACHTUNG - BITTE VORMERKEN:

Kongress Osteopathische Medizin 2015

06.-08.11.2015 in Mannheim

Positionen - Schnittstellen - Perspektiven 